\title{
Immunoreaction of 14F7 Mab Raised against N-Glycolyl GM3 Ganglioside Correlates with High Histological Grade in some Tumors of Neuroectodermal and Epithelial Lineage
}

Rancés Blanco ${ }^{1 *}$, Damián Blanco ${ }^{2}$, Xiomara Escobar², Charles E. Rengifo ${ }^{3}$, Mercedes Cedeñoํ․ Rosa Irene Alvarez ${ }^{2}$, Enrique Rengifo ${ }^{1}$ and Adriana Carr ${ }^{4}$

${ }^{1}$ Laboratory of Recognition and Biological Activity Assays, Department of Quality Control, Center of Molecular Immunology, Havana, Cuba

${ }^{2}$ Department of Cell Biology and Tissues Banking, National Institute of Oncology and Radiobiology, Havana, Cuba

${ }^{3}$ Department of Pathology, Manuel Fajardo General Hospital, Havana, Cuba

${ }^{4}$ Biomarkers Group, Research and Development Direction, Center of Molecular Immunology, Havana, Cuba

\begin{abstract}
The aberrant expression of $\mathrm{N}$-glycolyl GM3 ganglioside (NeuGcGM3) has been reported in a variety of malignant tumors. Nevertheless, the relationship between NeuGcGM3 expression and aggressive biological behavior still remains unclear for the majority of malignancies. In this article the tissue reactivity of the 14F7 monoclonal antibody, a highly specific IgG1 against NeuGcGM3, in breast cancer, urinary bladder tumors and malignant gliomas of adult patients is shown as well as its relation with the histological grade of these malignancies. The expression of NeuGcGM3 was detected in 92/155 (59.3\%) of tumors independently of the histopathological classification. However, a preferential expression of NeuGcGM3 was detected in: infiltrating ductal carcinoma $(77.1 \%)$ vs. infiltrating lobular carcinoma $(15.9 \%)(p=0.024)$, grade III $(94.9 \%)$ vs. grade II $(77.8 \%)$ and grade I $(63.8 \%)$ transitional cell carcinoma $(p=0.042)$ and high-grade astrocytomas $(78.6 \%)$ vs. low-grade astrocytic tumors $(10.0 \%)(p=0.026)$. The results achieved suggest the relationship between the tissue expression of NeuGcGM3 and the more aggressive biological behavior of these malignancies, regardless of the tumor cell lineage. The data obtained also support the continuous use of NeuGcGM3 as a target for immunotherapy in malignancies expressing this molecule as well as that of $14 \mathrm{~F} 7$ monoclonal antibody for the selection of candidate patients for these specific therapies.
\end{abstract}

Keywords: N-glycolyl GM3 ganglioside; 14F7 Mab; Tissue reactivity; Neuroectodermal and epithelial tumors; High histological grade

Abbreviations: CD: Cluster of Differentiation; CMP-NeuAc Hydroxylase: Cytidine Monophospho-N-Acetylneuraminic Acid Hydroxylase; DAB: 3,3-Diaminobencidine; DCs: Dendritic Cells; GT: Glycosyltransferase; IDC: Infiltrating Ductal Carcinoma; IgG1: Immunoglobulin Subclasse 1; ILC: Infiltrating Lobular Carcinomas; LSAB:Labelled-Streptavidin Biotin System; Mab:Monoclonal Antibody; NeuAc: N-Acetylneuraminic Acid; NeuGc: N-Glycolylneuraminic Acid; NeuGcGM3: N-Glycolyl GM3 Ganglioside; NOS: No Otherwise Specified; OMRS: Other Minorly Represented Subtype; STMR: Subtypes Minor Represented; TMA: Tissue Micro Arrays; VSSP: Very Small Size Proteoliposomes

\section{Introduction}

Gangliosides are sialic acid-bearing glycosphingolipids engaged in a variety of biological events that occur at vertebrate's cell membrane [1-3]. During malignant transformation, the composition of gangliosides changes at both quantitative and qualitative levels. Among the molecules contributing to tumor-associated carbohydrate structures of glycosphingolipids, sialic acid is considered one of the most important $[4,5]$.

The two major sialic acid variants in mammals are $\mathrm{N}$-acetylneuraminic acid (NeuAc) and N-glycolylneuraminic acid (NeuGc). NeuAc is the biosynthetic precursor of NeuGc, which is a component of gangliosides in most animal species. The conversion of NeuAc to NeuGc is catalyzed by the cytidine monophospho-NeuAc hydroxylase enzyme. However, in humans this enzyme is inactive [6]. In this sense, the aberrant expression of the NeuGc residue in humans has been associated with its incorporation from dietary sources to the altered metabolism of malignant cells [4,7], also favored by the hypoxic conditions of tumors [8].
Unusual glycolylated gangliosides have been identified by immunohistochemistry in a variety of human malignancies. Specifically, N-glycolyl GM3 ganglioside (NeuGcGM3) has shown limited expression in human normal tissues; however, it has been detected in several tumors [9-14] by means of $14 \mathrm{~F} 7$ monoclonal antibody (Mab) reactivity. This Mab is a mouse IgG1 specific for NeuGcGM3 [15]. As a consequence, NeuGcGM3 has become an attractive target for both active and passive immunotherapy of these malignancies.

Interestingly, in vitro and in vivo models demonstrated the immunosuppressive effects of NeuGcGM3 as well as its contribution to tumor cells progression [16-18]. In line with these results, the expression of NeuGcGM3 was associated with a more aggressive disease in colon adenocarcinoma [19] and non-small cell lung cancer [20]. However, opposite results were also reported [21,22]. In addition, differential expression of NeuGcGM3 has been detected in relation with the histological classification of tumors $[10,23]$. Nevertheless, the biological significance of this ganglioside still remains unclear for the majority of malignant tumors.

*Corresponding authors: Rancés Blanco. Center of Molecular Immunology. 216 Street and 15 Avenue, Atabey, Playa. PO Box 16040, Havana 11600; Cuba, Tel: (537) 2143133; Fax: (537) 2720644; E-mail: rances@cim.sld.cu

Received September 30, 2015; Accepted October 28, 2015; Published October 30, 2015

Citation: Blanco R, Blanco D, Escobar X, Rengifo CE, Cedeño M, et al. (2015) Immunoreaction of 14F7 Mab Raised against N-Glycolyl GM3 Ganglioside Correlates with High Histological Grade in some Tumors of Neuroectodermal and Epithelial Lineage. J Mol Biomark Diagn 6: 252. doi:10.4172/2155-9929.1000252

Copyright: $\odot 2015$ Blanco R, et al. This is an open-access article distributed under the terms of the Creative Commons Attribution License, which permits unrestricted use, distribution, and reproduction in any medium, provided the original author and source are credited. 
Citation: Blanco R, Blanco D, Escobar X, Rengifo CE, Cedeño M, et al. (2015) Immunoreaction of $14 \mathrm{~F} 7$ Mab Raised against N-Glycolyl GM3 Ganglioside Correlates with High Histological Grade in some Tumors of Neuroectodermal and Epithelial Lineage. J Mol Biomark Diagn 6: 252. doi:10.4172/2155-9929.1000252

Page 2 of 6

In the present work the tissue expression of NeuGcGM3 in breast cancer, urinary bladder tumors and malignant gliomas of adult patients as well as its relation with the histological grade of these neoplasms is shown.

\section{Materials and Methods}

\section{Monoclonal antibody}

14F7 Mab (IgG1), a highly specific anti-NeuGcGM3 ganglioside antibody, produced by the Center of Molecular Immunology (Havana, Cuba) was used. This Mab was generated with the methodology previously described in ref. [15]. 14F7 Mab is able to detect the carbohydrate antigenic determinant of NeuGcGM3 ganglioside after formalin fixation and routine tissue processing [20,24].

\section{Study design}

A retrospective, observational study of a consecutive series of patients diagnosed with breast cancer $(n=46)$, urinary bladder malignant tumors $(n=55)$ and malignant gliomas $(n=54)$ was performed. All tissue samples were received from the pathology departments of both National Institute of Oncology and Radiobiology (Havana, Cuba) and the National Institute of Neurology and Neuropathology (Havana, Cuba), after obtaining approved consent from the institutional ethical committees.

\section{Tissue specimens and previous processing}

For urinary bladder tumors, tissue micro arrays (TMA) were made. Briefly, each block of paraffin-embedded tumor material was cut into $5 \mu \mathrm{m}$-thick sections and placed on glass slides (Dako, S2024). Slides were stained with Hematoxylin and eosin and an expert pathologist verified the presence of tumor cells and marked the tumor area. Two $\mathrm{mm}$ diameter cylinders were taken from each block and they were included in recipient tissue array blocks using a precision tissue array instrument (Thermo Scientifics, TMA-001).

For all samples, five micrometer serial sections from each paraffin block were obtained in a microtome (Lizt 1512, Germany). All sections were attached to the slides by heating at $60^{\circ} \mathrm{C}$ in oven for 1 hour. Afterward, the slides were kept at room temperature until they were used. The slides were dewaxed in xylene and rehydrated in graded ethanol series following the commonly used procedure for this operation and endogenous peroxidase activity was blocked with the peroxidase-blocking reagent (Dako, K5207) for 10 minutes. All sections were rehydrated in distilled water for 10 minutes and then rinsed with wash buffer (Dako, K5207).

\section{Immunohistochemical staining}

Slides were placed in a humid chamber and incubated with $14 \mathrm{~F} 7$ $\mathrm{Mab}$ for $1 \mathrm{~h}$ at room temperature. Negative controls were performed substituting the primary antibody for the washing buffer. Sections of ductal breast carcinoma were taken as positive control [11].

After two rinses in wash buffer, the slides were incubated with the reagents of LSAB detection system (Dako, K0690) for 30 minutes in each step. Between incubations, samples were washed with wash buffer for 10 minutes. Enzymatic activity was visualized with DAB substrate chromogen solution (Dako K3465). Slides were counterstained with Mayer's Hematoxylin (Dako S2020), dehydrated and mounted with a synthetic medium.

\section{Evaluation of results}

The intensity of the reaction of each sample was qualitatively estimated and expressed as follows: negative (0), weak (1), moderate
(2) and intense (3). Then, in each specimen, the percentage of positive tumor cells in the most representative areas was measured using a 100X magnification. Results obtained by two independent observers were taken to do the final evaluation of the analysis. Afterward, the percentage of positive cells and the intensity of reaction were multiplied in each specimen, resulting in a score ranging from 0 to 300. Finally, these scores were classified in this manner: low expression (scores $<150$ ) and high expression (scores $\geq 150$ ) to obtain the final score (F-score) as previously described in ref. [20].

\section{Statistical analysis}

GraphPad Prism 5 software (2007 GraphPad Software Inc. La Jolla, CA USA) was used for data analysis. The relation between 14F7 Mab immunoreactivity and histological grade of malignant tumors was analyzed using the Chi-square test. Correlations were assessed by Spearman ranks correlation coefficients. A p value $<0.05$ was considered statistically significant.

\section{Results}

\section{Immunohistochemical localization of NeuGcGM3}

In general, the reactivity of $14 \mathrm{~F} 7 \mathrm{Mab}$ was observed on both plasmatic membrane and cytoplasm of malignant cells with a homogeneous and finely granular pattern of staining as previously described in ref. [24]. According to the F-score, the expression of NeuGcGM3 was detected in 92/155 (59.3\%) of tumors independently of the histopathological classification.

\section{Breast cancer}

A weak to intense reactivity with $14 \mathrm{~F} 7 \mathrm{Mab}$ was observed in 45/46 (97.8\%) of breast carcinomas, regardless of the histological subtype of tumors (Table 1). Only, one case of infiltrating lobular carcinoma was not reactive to this Mab. In all positive cases, the expression of NeuGcGM3 was observed in more than $95 \%$ of malignant epithelial cells. However, only 31/46 (67.4\%) of breast carcinomas showed high levels of NeuGcGM3 expression. The staining of 14F7 Mab according to the histological subtype of tumors followed this distribution: 13/15 (86.7\%) of no otherwise specified (NOS) infiltrating ductal carcinomas (IDC), 14/20 (70.0\%) of other minorly represented subtype (OMRS) infiltrating ductal carcinomas and $4 / 11$ (15.9\%) of infiltrating lobular carcinomas (ILC).

A preferential expression of this ganglioside was detected in IDC when compared with ILC ( $77.1 \%$ vs $15.9 \%)$. A statistically significant difference was evidenced when the immunoreaction with 14F7 Mab in these tumors was compared ( $\mathrm{p}=0.024$; Chi-square test) (Figure 1). But, no difference was obtained when the expression of NeuGcGM3 was compared in NOS infiltrating ductal carcinomas and OMRS infiltrating ductal carcinomas.

\section{Urinary bladder tumors}

The tissue expression of NeuGcGM3 was observed in 49/55 (89.1\%) cases and different intensities of reaction and percentage of positive cells were obtained. However, according to the F-score 46/55 (83.6\%) of urinary bladder tumors showed high levels of 14F7 Mab staining (Table 1). Compared with other urinary bladder tumors, transitional cell carcinoma exhibited increased levels of NeuGcGM3 expression in $45 / 53(84.9 \%)$ cases without taking into consideration the degree of cellular atypia gradation.

The tissue expression of NeuGcGM3 increased progressively together with the histological grade of tumors $63.6 \%(7 / 11), 77.8 \%(7 / 9)$ 
Citation: Blanco R, Blanco D, Escobar X, Rengifo CE, Cedeño M, et al. (2015) Immunoreaction of $14 \mathrm{~F} 7$ Mab Raised against N-Glycolyl GM3 Ganglioside Correlates with High Histological Grade in some Tumors of Neuroectodermal and Epithelial Lineage. J Mol Biomark Diagn 6: 252. doi:10.4172/2155-9929.1000252

Page 3 of 6

\begin{tabular}{|l|c|c|c|}
\hline \multirow{2}{*}{ Histopathological classification } & \multicolumn{2}{|c|}{ 14F7 Mab reactivity } & \multirow{2}{*}{$\begin{array}{c}\text { Positive } \\
\text { cases (\%) }\end{array}$} \\
\cline { 2 - 3 } & Low & High & \\
\cline { 2 - 3 } Breast cancer & & & \\
\hline Infiltrating lobular carcinoma & 7 & 4 & 15.9 \\
\hline Infiltrating ductal carcinoma & & & \\
\hline No otherwise specified (NOS) & 2 & 13 & 86.7 \\
\hline Other subtype minor represented & 6 & 14 & 70.0 \\
\hline Total & 15 & 31 & 67.4 \\
\hline Urinary bladder tumors & & & \\
\hline Urothelial cell carcinoma & & & \\
\hline Grade I & 4 & 7 & 63.6 \\
\hline Grade II & 2 & 7 & 77.8 \\
\hline Grade III & 2 & 31 & 93.9 \\
\hline Squamous cell carcinoma & 0 & 1 & 100 \\
\hline Undifferentiated tumor & 1 & 0 & 0 \\
\hline Total & 9 & 46 & 83.6 \\
\hline Malignant gliomas & & & \\
\hline Astrocytomas & & & \\
\hline Grade I & 4 & 0 & 0.0 \\
\hline Grade II & 5 & 1 & 16.7 \\
\hline Grade III & 9 & 4 & 30.8 \\
\hline Grade IV & 5 & 7 & 58.3 \\
\hline Other low-grade gliomas & 16 & 3 & 15.8 \\
\hline Total & 39 & 15 & 27.8 \\
\hline Legend $\%$, pernnn \\
\hline
\end{tabular}

Legend. \%, percentage; F-score: Low expression of NeuGcGM3 (F-score<150); High expression of NeuGcGM3 (F-score $\geq 150$ ).

Table 1: Immunoreaction of 14F7 Mab according to the histological classification of malignant tumors

and $93.9 \%$ (31/33) for grade I, II and III transitional cell carcinoma, respectively. Although, only a statistical trend was obtained $(\mathrm{p}=0.079$, Spearman coefficient $=0.260$; Spearman Test). A statistically significant association was evidenced when the expression of NeuGcGM3 was compared in grade I, II and grade III transitional cell carcinoma $(\mathrm{p}=0.042$; Chi-square test $)$ (Figure 2).

\section{Malignant gliomas}

In general, high levels of NeuGcGM3 expression was evidenced in $15 / 54(27.8 \%)$ of all malignant gliomas (Table 1). Low-grade astrocytoma (I-II) showed a limited reactivity with 14F7 Mab (1/10). But, the recognition of 14F7 Mab gradually was associated with the progressive increase in the malignancy of malignant astrocytomas with $0.0 \%(0 / 4), 16.7 \%(1 / 6), 30.8 \%(4 / 13)$ and $58.3 \%(7 / 12)$ of positive cases for grade I, II, III and IV astrocytomas, respectively ( $\mathrm{p}=0.013$, Spearman coefficient $=0.414$; Spearman Test). Consequently, the reactivity of $14 \mathrm{~F} 7$ Mab was greater in high-grade astrocytomas as compared to low-grade tumors ( $\mathrm{p}=0.026$; Chi-square Test).

In addition, the reaction with 14F7 Mab was evidenced in 3/19 (15.8\%) cases of other low-grade gliomas. A statistically significant difference was obtained when the staining with this Mab was compared in other low-grade gliomas and high-grade astrocytomas $(\mathrm{p}=0.015$; Chi-square Test) (Figure 3). However no statistically significant difference was evidenced between the expression of NeuGcGM3 in low-grade astrocytomas and other low-grade gliomas.

\section{Discussion}

Changes in the cell surface glycolipids composition that take place during malignant transformation have been previously described

[5]. The appearance of specific carbohydrate epitopes in certain tumors have been reported to affects their progression, invasive and metastatic potential $[25,26]$. In particular, some studies have shown the relevance of NeuGcGM3 in cancer progression. NeuGcGM3 appeared to be involved in tumor-induced DC suppression [21], in the downmodulation of CD4 expression in T lymphocytes [16] as well as, in the proliferation and differentiation of $\mathrm{CD} 4^{+} \mathrm{CD} 25^{-} \mathrm{T}$ lymphocytes [17], abrogating an anti-tumor-specific immune response.

Breast tumour tissues were shown to be distinct from normal mammary tissues in terms of both ganglioside and glycosyltransferase (GT) genes composition. The gangliosides GM3 (the $\mathrm{N}$-acetyl variant), GD3 and the derivatives 9-O-acetyl-GD3 (CDw60 antigen) and 9-O-acetyl-GT3, which show a very restricted expression in normal breast tissues, are over-expressed in IDC [27]. It was also suggested that GM3 or GD3 synthase (ST8Sia I) overexpression may contribute to increasing the malignant properties of breast cancer cells by mediating cell proliferation and migration [28]. ST8Sia I over-expression was associated with both poor histopathological grading in oestrogen receptor-negative tumors and lower survival of patients [29].

Marquina et al. reported that the expression of NeuGcGM3 in breast tumors using mass spectrometry analysis [27]. Later, Carr et al. reported the generation of 14F7 Mab, a highly specific IgG against NeuGcGM3 that recognizes breast infiltrating ductal carcinoma and cutaneous melanoma by immunohistochemistry on both frozen [15] and formalin-fixed and paraffin embedded samples [11,14]. The ability of $14 \mathrm{~F} 7 \mathrm{Mab}$ labelled with $99 \mathrm{mTc}$ to recognize breast tumors in vivo by the radioimmunoscintigrafic technique was also demonstrated [30]. Nevertheless, no information about the potential role of NeuGcGM3 in the biological behavior of breast cancer has been found in the literature consulted.

In this paper, differences in the levels of NeuGcGM3 expression according to the histological subtype of breast carcinoma are reported for the first time. These results were obtained by means of the application of a novel score that integrates the percentage of 14F7-positive cells with the intensity of the immunohistochemical reaction (F-score) [20] A preferential expression of NeuGcGM3 was evidenced in IDC as compared with ILC, indicating a more aggressive biological behavior
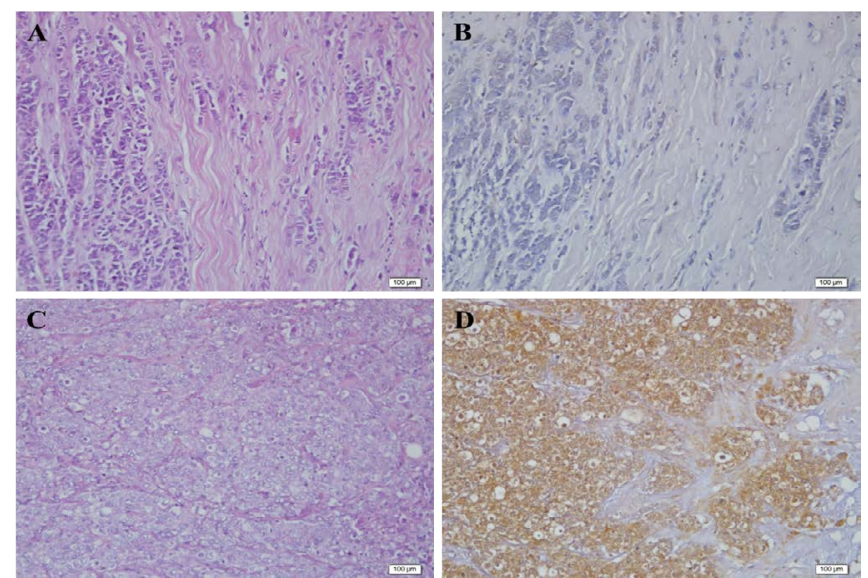

Figure 1: Immunoreactiviy of 14F7 Mab in breast cancer. $\mathrm{A}$ and $\mathrm{C}$ : Hematoxylin and eosin staining of infiltrating lobular and ductal carcinomas, respectively. Observe: the very weak expression of NeuGcGM3 in the infiltrating lobular carcinoma section $(B)$ while an intense reaction with 14F7 Mab was evidenced in infiltrating ductal carcinoma (D) (Brown color). Counterstaining with Mayer's Hematoxylin (Blue color). White bar $=100 \mu \mathrm{m}$. 
Citation: Blanco R, Blanco D, Escobar X, Rengifo CE, Cedeño M, et al. (2015) Immunoreaction of $14 \mathrm{~F} 7$ Mab Raised against N-Glycolyl GM3 Ganglioside Correlates with High Histological Grade in some Tumors of Neuroectodermal and Epithelial Lineage. J Mol Biomark Diagn 6: 252. doi:10.4172/2155-9929.1000252

Page 4 of 6
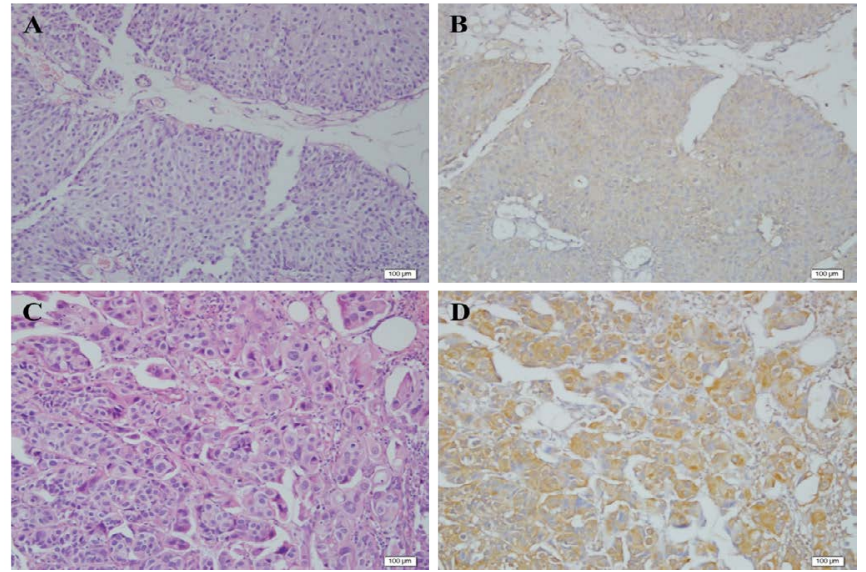

Figure 2: Immunostaining of $14 \mathrm{~F} 7 \mathrm{Mab}$ in urinary bladder tumors. A and C: Hematoxylin and eosin staining of grade I and grade III transitional cell carcinomas, respectively. Observe: a low expression of NeuGcGM3 (Brown color) in the grade I transitional cell carcinoma sample (B). By the contrary, high level of this ganglioside was detected in grade III tumor (D). Counterstaining with Mayer's Hematoxylin (Blue color). White bar $=100 \mu \mathrm{m}$.

of these malignancies. In line with these results, it was demonstrated that the metastatic potential and the stage-matched prognosis is worse for patients with IDC than ILC [31].

In a previous work, Blanco et al. reported the expression of NeuGcGM3 in lymph node metastasis of breast cancer as well as its incorporation to the cell membrane of peritumoral lymphocytes [14]. A clear association between NeuGcGM3 insertion in lymphocyte plasma membranes and the CD4 down-modulation effect was also documented [16]. In this way, our results and those concerning to the high expression of NeuGcGM3 in lymph node metastasis of breast infiltrating carcinoma suggest that higher levels of this ganglioside could contribute to a more accelerated cancer progression and metastasis of IDC. In line with this, the expression of ST6GalNAc V, a sialyltransferase involved in the biosynthesis of $\alpha$-series gangliosides, enhances the adhesion of breast cancer metastatic cells to brain endothelium and their passage through the blood-brain barrier [32].

Differential patterns of gangliosides expression and distribution have been also found in malignant bladder tumors [33,34]. Blanco et al. previously reported a preliminary study of NeuGcGM3 expression in transitional cell carcinoma $(n=9)$ [11]. In the present work, the study of the reactivity of 14F7 Mab was extended to 55 cases of bladder tumors. The expression of NeuGcGM3 was evidenced in the $83.6 \%$ of them, independently of the histological grade. Interestingly, it was revealed a direct correlation between the amounts of NeuGcGM3 expressed and the histological grade of these malignancies. In fact, the $14 \mathrm{~F} 7$ Mab staining was significantly increased in grade III transitional cell carcinoma tumors as compared with grade I-II.

Todeschini et al. reported the relation of GM2, GM3 and/or GM2/ GM3 complexes in cell motility and growth in bladder cancer [33]. In addition, over-expression of GM3 induces apoptosis and reduces the malignant potential in murine bladder cancer [33]. Interestingly, a greater presence of GM3 in nonmuscle invasive papillary tumors of urinary bladder was detected as compared with invasive tumors [35]. Furthermore, GM3 diminishes the cancer cell proliferation by inhibiting EGFR tyrosine kinase [36] and modulating the expression of cell cycle regulation proteins [37]. However, NeuGcGM3 variant is not able to inhibit the EGFR tyrosine kinase as much as the
$\mathrm{N}$-acetylated GM3 variant [38]. Aberrant accumulation of NeuGcGM3 in transitional cell carcinoma could diminish the anti-tumor effects of GM3, contributing to the potential invasion of these tumors. In this sense, our results suggest that the amount of NeuGcGM3 expressed may serve as an indicator of the invasive potential of bladder tumors.

Recently, Scursoni et al. reported the expression of NeuGcGM3 in pediatric neuroblastoma using formalin-fixed and paraffin-embedded tissues [39]. Also, the 14F7 Mab reaction was demonstrated by Blanco et al. in both pediatric and adult gliomas, although the number of adult gliomas samples evaluated in that study was limited $(n=8)$ [13]. In this study, the expression of NeuGcGM3 was detected in about $30 \%$ of malignant gliomas of adult patients regardless of the histological grade of tumors. Nevertheless, malignant gliomas expressing higher levels of NeuGcGM3 were preferably high-grade tumors rather low-grade tumors.

It is known that the progression of malignant brain tumors is associated with altered gangliosides composition and distribution. The literature reports a decrease in the total amount of more complex gangliosides (polysialylated) joint to increased proportions of simpler mono-and disialogangliosides, such as GM3, GM2, GD3 and GD2 [4042]. GM3 is able to inhibit angiogenesis, proliferation and invasion of glioma cells $[41,43]$. The accumulation of GM3 has been evidenced in a variety of brain malignancies [40,42]. Nevertheless, the preferential accumulation of NeuGcGM3 in high-grade gliomas seems to be in agreement with the more aggressive biological behavior of these tumors. In this sense, our results are consistent with a previous report of Scursoni et al., who found a correlation between the expression of NeuGcGM3 with a more aggressive form of pediatric neuroblastomas [39].

Tumor hypoxia has been considered one of the most important causes of both the aggressive course of malignant gliomas and the resistance to conventional therapies. Malignant glioma cells produce a number of different immunosuppressive mediators that inhibit antitumor immunity, contributing to their rapid proliferation even in a hypoxic environment $[8,44]$. Tumor hypoxia is considered responsible of NeuGcGM2 ganglioside expression in human cancer cells through the incorporation of NeuGc. The effect of hypoxia could be to expedite
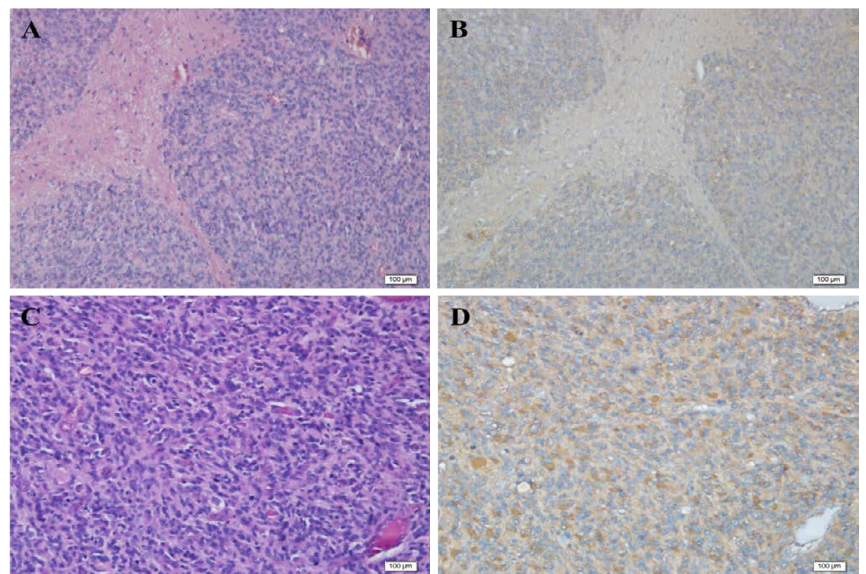

Figure 3: Immunoreaction of $14 \mathrm{~F} 7 \mathrm{Mab}$ in malignant gliomas. $\mathrm{A}$ and $\mathrm{C}$ : Hematoxylin and eosin staining of ependimoma (grade II) and glioblastoma (grade IV), respectively. Observe: a weak reactivity of 14F7 Mab in ependimoma section (Brown color). Nevertheless, an increased expression of NeuGcGM3 was evidenced in the glioblastoma cells (D). Counterstaining with Mayer's Hematoxylin (Blue color). White bar=100 $\mu \mathrm{m}$. 
Citation: Blanco R, Blanco D, Escobar X, Rengifo CE, Cedeño M, et al. (2015) Immunoreaction of 14F7 Mab Raised against N-Glycolyl GM3 Ganglioside Correlates with High Histological Grade in some Tumors of Neuroectodermal and Epithelial Lineage. J Mol Biomark Diagn 6 : 252. doi:10.4172/2155-9929.1000252

Page 5 of 6

sialic acid transport from the external medium, due to the increase observed in sialin expression (a sialic acid molecule transporter) [8]. Moreover, higher levels of NeuGcGM2 and NeuGcGM3 gangliosides were detected in U87-MG glioblastoma cells that grew as a xenograft in severe combined immunodeficiency (SCID) mice (in vivo) as compared to these cells in cultures (in vitro) [45]. Further experiments in order to explore the potential relation between tumor hypoxia and the expression of NeuGcGM3 in malignant gliomas are being planned by our group.

In Cuba, some clinical trials have been performed for the treatment of cancer patients using two immunotherapeutic approaches targeting NeuGcGM3: a molecular vaccine (NeuGcGM3/VSSP) [46] and an anti-idiotype antibody-based vaccine (racotumomab, 1E10) [47]. Preliminary results suggested that racotumomab and NeuGcGM3/ VSSP induce immune response to NeuGcGM3 antigen, improving the survival of treated patients [46,47]. However, other immune mechanisms could be involved in this survival benefits. Additionally, other clinical trials are currently being designed with a humanized version of $14 \mathrm{~F} 7 \mathrm{Mab}$. In preclinical scenarios, this Mab was able to induce potent antitumor activity both in vitro and in vivo on a solid mouse myeloma model $[48,49]$. The data obtained in this study could permit to consider the design of newer clinical trials with these immunotherapeutic agents in urinary bladder tumors and malignant gliomas that express NeuGcGM3, along or combined with the established modalities of treatment.

In summary, we confirmed the presence of NeuGcGM3 ganglioside in breast cancer, urinary bladder tumors and malignant gliomas. Interestingly, increased expression of NeuGcGM3 is correlated with the high histological grade in these malignancies. Moreover, the preferential expression of this ganglioside accompanied the disease progression in urinary bladder neoplasms and malignant astrocytomas. Our results suggest the relationship between the tissue expression of NeuGcGM3 and the more aggressive biological behavior of these malignancies, regardless of the tumor cell lineage. Our data also support the continuous use of NeuGcGM3 as a target for immunotherapy of malignancies expressing this molecule as well as the use of the murine version of 14F7 Mab for the selection of candidate patients for these specific therapies.

\section{Acknowledgement}

The authors want to express grateful to B.Sc. Miriela Soto as well as to M.Sc Rolando Dominguez for the editorial assistance. Financial support was provided by the Center of Molecular Immunology, Havana, Cuba.

\section{References}

1. Doronin II, Vishnyakova PA, Kholodenko IV, Ponomarev ED, Ryazantsev DY et al. (2014) Ganglioside GD2 in reception and transduction of cell death signal in tumor cells. BMC Cancer 14: 295.

2. Jales A, Falahati R, Mari E, Stemmy EJ, Shen W, et al. (2011) Gangliosideexposed dendritic cells inhibit T-cell effector function by promoting regulatory cell activity. Immunology 132: 134-143.

3. Shimada S, Ito A, Kawasaki Y, Kakoi N, Taima T, et al. (2014) Ganglioside disialosyl globopentaosylceramide is an independent predictor of PSA recurrence-free survival following radical prostatectomy. Prostate Cancer Prostatic Dis 17: 199-205.

4. Samraj AN, Läubli H, Nissi Varki N, Varki A (2014) Involvement of a non-human sialic acid in human cancer. Front Oncol 4:33.

5. Don AS, Lim XY, Couttas TA (2014) Re-configuration of sphingolipid metabolism by oncogenic transformation. Biomolecules 4: 315-353.

6. Irie A, Koyama S, Kozutsumi Y, Kawasaki T, Suzuki A (1998) The molecular basis for the absence of $\mathrm{N}$-glycolylneuraminic acid in humans. J Biol Chem 273: $15866-15871$
7. Varki A (2001) N-glycolylneuraminic acid deficiency in humans. Biochimie 83: 615-622.

8. Yin J, Miyazaki K, Shaner RL, Merrill AH Jr, Kannagi R (2010) Altered sphingolipid metabolism induced by tumor hypoxia - new vistas in glycolipid tumor markers. FEBS Lett 584: 1872-1878.

9. Blanco R, Rengifo E, Cedeño M, Rengifo CE, Alonso DF, et al. (2011) Immunoreactivity of the 14F7 Mab Raised against N-Glycolyl GM3 Ganglioside in Epithelial Malignant Tumors from Digestive System. ISRN Gastroenterol 645641.

10. Blanco R, Rengifo E, Rengifo CE, Cedeño M, Frómeta M, et al. (2011) Immunohistochemical Reactivity of the 14F7 Monoclonal Antibody Raised against N-Glycolyl GM3 Ganglioside in Some Benign and Malignant Skin Neoplasms. ISRN Dermatol 848909.

11. Blanco R, Cedeño M, Escobar X, Blanco D, Rengifo CE, et al. (2011) Immunorecognition of the 14F7 Mab raised against N-glycolyl GM3 ganglioside in some normal and malignant tissues from genitourinary system. ISRN Pathol 953803

12. Blanco R, Rengifo CE, Cedeño M, Frómeta M, Rengifo E, et al. (2012) Immunoreactivity of the 14F7 Mab (Raised against N-Glycolyl GM3 Ganglioside) as a Positive Prognostic Factor in Non-Small-Cell Lung Cancer. Patholog Res Int 235418 .

13. Blanco R, Quintana Y, Blanco D, Cedeño M, Rengifo CE, et al. (2013) Tissue Reactivity of the 14F7 Mab Raised against N-Glycolyl GM3 Ganglioside in Tumors of Neuroectodermal, Mesodermal, and Epithelial Origin. J Biomark 602417

14. Blanco R, Blanco D, Quintana Y, Escobar X, Rengifo CE, et al. (2013) Immunoreactivity of the 14F7 Mab Raised against N-Glycolyl GM3 Ganglioside in Primary Lymphoid Tumors and Lymph Node Metastasis. Patholog Res Int 920972.

15. Carr A, Mullet A, Mazorra Z, Vázquez AM, Alfonso M, et al. (2000) A mouse IgG1 monoclonal antibody specific for $\mathrm{N}$-glycolyl GM3 ganglioside recognized breast and melanoma tumors. Hybridoma 19: 241-247.

16. de Leòn J, Fernández A, Mesa C, Clavel M, Fernández LE (2006) Role of tumour-associated $\mathrm{N}$-glycolylated variant of GM3 ganglioside in cance progression: effect over CD4 expression on $\mathrm{T}$ cells. Cancer Immunol Immunother 55: 443-450

17. de León J, Fernández A, Clavell M, Labrada M, Bebelagua $Y$, et al. (2008) Differential influence of the tumour-specific non-human sialic acid containing GM3 ganglioside on CD4+CD25- effector and naturally occurring CD4+CD25+ regulatory T cells function.Int Immunol 20: 591-600.

18. Casadesús AV, Fernández-Marrero $\mathrm{Y}$, Clavell M, Gómez JA, Hernández T et al. (2013) A shift from N-glycolyl- to N-acetyl-sialic acid in the GM3 ganglioside impairs tumor development in mouse lymphocytic leukemia cells. Glycoconj J 30: 687-99.

19. Lahera T, Calvo A, Torres G, Rengifo CE, Quintero S, et al. (2014) Prognostic Role of 14F7 Mab Immunoreactivity against N-Glycolyl GM3 Ganglioside in Colon Cancer. J Oncol 2014: 482301.

20. Blanco R, Domínguez E, Morales O, Blanco D, Martínez D, et al. (2015) Prognostic significance of N-Glycolyl GM3 ganglioside expression in non-smal cell lung carcinoma patients: new evidences. Pathol Res Int 132326 (In press)

21. Van Cruijsen H, Gallegos Ruiz M, Van der Valk P, D de Gruijl T, Giaccone G (2009) Tissue micro array analysis of ganglioside N-glycolyl GM3 expression and signal transducer and activator of transcription (STAT)-3 activation in relation to dendritic cell infiltration and micro vessel density in non-small cell lung cancer. BMC Cancer 9: 180

22. Zhong Y, Wu Y, Li C, Tang J, Wang X, et al. (2012) N-Glycolyl GM3 ganglioside immunoexpression in oral mucosal melanomas of Chinese. Oral Dis 18: 741 747.

23. Scursoni AM, Galluzzo L, Camarero S, Pozzo N, Gabri MR, et al. (2010) Detection and characterization of $\mathrm{N}$-glycolyated gangliosides in Wilms tumor by immunohistochemistry. Pediatr Dev Pathol 13: 18-23.

24. Blanco R, Rengifo ChE, Cedeño M, Frómeta M, Hernández T, et al. (2014) Immunodetection of $\mathrm{N}$-glycolyl GM3 ganglioside in lung carcinoma by immunohistochemistry: a technical study using frozen and formalin-fixed and paraffin-embedded tissues. Acta Microsc 23: 199-213. 
Citation: Blanco R, Blanco D, Escobar X, Rengifo CE, Cedeño M, et al. (2015) Immunoreaction of 14F7 Mab Raised against N-Glycolyl GM3 Ganglioside Correlates with High Histological Grade in some Tumors of Neuroectodermal and Epithelial Lineage. J Mol Biomark Diagn 6 : 252. doi:10.4172/2155-9929.1000252

Page 6 of 6

25. Pochechueva T, Jacob F, Fedier A Heinzelmann-Schwarz V (2012) TumorAssociated Glycans and Their Role in Gynecological Cancers: Accelerating Translational Research by Novel High-Throughput Approaches. Metabolites 2 : 913-939.

26. Tuccillo FM, de Laurentiis A, Palmieri C, Fiume G, Bonelli P, et al. (2014) Aberrant glycosylation as biomarker for cancer: focus on CD43. Biomed Res Int 2014: 742831

27. Marquina G, Waki H, Fernández LE, Kon K, Carr A, et al. (1996) Gangliosides expressed in human breast cancer. Cancer Res 56: 5165-5171.

28. Cazet A, Julien S, Bobowski M, Burchell J, Delannoy P (2010) Tumourassociated carbohydrate antigens in breast cancer. Breast Cancer Res 12: 204

29. Ruckhäberle E, Karn T, Rody A, Hanker L, Gätje R, et al. (2009) Gene expression of ceramide kinase, galactosyl ceramide synthase and ganglioside GD3 synthase is associated with prognosis in breast cancer. J Cancer Res Clin Oncol 135: 1005-1013

30. Oliva JP, Valdés Z, Casacó A, Pimentel G, González J, et al. (2006) Clinical evidences of GM3 (NeuGc) ganglioside expression in human breast cance using the 14F7 monoclonal antibody labelled with 99mTc. Breast Cancer Res Treat 96: 115-121.

31. Wasif N, Maggard MA, Ko CY, Giuliano AE (2010) Invasive lobular vs. ducta breast cancer: a stage-matched comparison of outcomes. See comment in PubMed Commons below Ann Surg Oncol 17: 1862-1869.

32. Bos PD, Zhang XH, Nadal C, Shu W, Gomis RR, et al. (2009) Genes that mediate breast cancer metastasis to the brain. Nature 459: 1005-1009.

33. Todeschini AR, Dos Santos JN, Handa K, Hakomori SI (2008) Ganglioside GM2/GM3 complex affixed on silica nanospheres strongly inhibits cell motility through CD82/cMet-mediated pathway. Proc Natl Acad Sci U S A 105: 19251930.

34. Kawamura S, Ohyama C, Watanabe R, Satoh M, Saito S, et al. (2001) Glycolipid composition in bladder tumor: a crucial role of GM3 ganglioside in tumor invasion. Int J Cancer 94: 343-347.

35. Watanabe R, Ohyama C, Aoki H, Takahashi T, Satoh M, et al. (2002) Ganglioside $\mathrm{G}(\mathrm{M} 3)$ overexpression induces apoptosis and reduces malignant potential in murine bladder cancer. Cancer Res 62: 3850-3854.

36. Bremer EG, Schlessinger J, Hakomori S (1986) Ganglioside-mediated modulation of cell growth. Specific effects of GM3 on tyrosine phosphorylation of the epidermal growth factor receptor. J Biol Chem 261: 2434-2340.
37. Hee-Jung C, Tae-Wook C, Sung-Koo K, Young-Choon L, Jeong-Heon K, et al. (2006) Ganglioside GM3 modulates tumor suppressor PTEN-mediated cell cycle progression-transcriptional induction of p21 WAF1 and p27 kip1 by inhibition of PI-3K/AKT pathway. Glycobiology 16: 573-583.

38. Hayashi N, Chiba H, Kuronuma K, Go S, Hasegawa Y, et al. (2013) Detection of $\mathrm{N}$-glycolyated gangliosides in non-small-cell lung cancer using GMR8 monoclonal antibody. Cancer Sci 104: 43-47.

39. Scursoni AM, Galluzzo L, Camarero S, Lopez J, Lubieniecki F, et al. (2011) Detection of $\mathrm{N}$-glycolyl GM3 ganglioside in neuroectodermal tumors by immunohistochemistry: an attractive vaccine target for aggressive pediatric cancer. Clin Dev Immunol 245181.

40. Shinoura N, Dohi T, Kondo T, Yoshioka M, Takakura K, et al. (1992) Ganglioside composition and its relation to clinical data in brain tumors. Neurosurgery 31 : 541-549.

41. Seyfried TN, Mukherjee P (2010) Ganglioside GM3 Is Antiangiogenic in Malignant Brain Cancer. J Oncol 961243.

42. Berra B, Gaini SM, Riboni L (1985) Correlation between ganglioside distribution and histological grading of human astrocytomas. Int J Cancer 36: 363-366.

43. Fujimoto Y, Izumoto S, Suzuki T, Kinoshita M, Kagawa N (2005) Ganglioside GM3 inhibits proliferation and invasion of glioma. J Neurooncol 71:99-106.

44. Perng P, Lim M (2015) Immunosuppressive Mechanisms of Malignant Gliomas: Parallels at Non-CNS Sites. Front Oncol 5: 153.

45. Ecsedy JA, Holthaus KA, Yohe HC, Seyfried TN (1999) Expression of mouse sialic acid on gangliosides of a human glioma grown as a xenograft in SCID mice. J Neurochem 73: 254-259.

46. Pérez K Osorio M, Hernández J, Carr A Fernández LE (2013) NGcGM3/ VSSP vaccine as treatment for melanoma patients. See comment in PubMed Commons below Hum Vaccin Immunother 9: 1237-1240.

47. Macias A, Alfonso S, Santiesteban E (2012) Active specific immunotherapy with racotumomab in the treatment of advanced non-small cell lung cancer. Ann Oncol 23(suppl 9).

48. Fernández-Marrero $Y$, Roque-Navarro $L$, Hernández $T$, Dorvignit $D$, MolinaPérez $M$, et al. (2011) A cytotoxic humanized anti-ganglioside antibody produced in a murine cell line defective of $\mathrm{N}$-glycolylated-glycoconjugates. Immunobiology 216: 1239-1247.

49. Dorvignit D, García-Martínez L, Rossin A, Sosa K, Viera J, et al. (2015) Antitumor and cytotoxic properties of a humanized antibody specific for the GM3(Neu5Gc) ganglioside. Immunobiology 220: 1343-1350. 